\title{
Impact of Imperfect CSI on Resource Allocation in Cognitive Radio Channels
}

\author{
Raouia Masmoudi( $^{(1)(2)}$, E. Veronica Belmega ${ }^{(1)(3)}$, Inbar Fijalkow ${ }^{(1)}$ \\ (1) ETIS, UMR 8051, University Paris Seine, University Cergy-Pontoise, ENSEA, CNRS, France \\ (2) LIGM, University Paris-Est Marne-la-vallée, France \\ (3) INRIA Grenoble - Rhône-Alpes, France \\ Emails:raouia.masmoudi@gmail.com, belmega@ensea.fr, inbar.fijalkow@ensea.fr
}

\begin{abstract}
Channel state information (CSI) is essential for efficient power and spectrum allocation policies. In cognitive radio (CR) channels, although perfect CSI of the direct link (between the secondary transmitter and the secondary receiver) is a reasonable assumption at the secondary transmitter (ST), however, perfect knowledge of its interfering links to the primary receivers (PRs) is not. Power allocation and scheduling algorithms are often based on perfect global CSI at the secondary transmitter (ST). In this paper, we analyze the impact of channel estimation errors on both the secondary and primary users. On the one hand, the robustness of water-filling type of algorithms allowing the secondary user ( $\mathrm{SU}$ ) to minimize its power consumption under QoS and CR interference power constraints to channel estimation errors in the SU interfering links is analyzed. On the other hand, the impact of these estimation errors on the PU interference constraints is also analyzed. To this aim, we consider the worst case with respect to these estimation errors. Our analysis shows that the water-filling algorithm provides robustness in terms of power consumption and scheduling of the $\mathrm{SU}$ given the realistic estimation error models especially when the $\mathrm{SU}$ is overestimating the interfering power gains. We also provide possible solutions to ensure that the created interference is below the tolerated thresholds.

Index Terms-Cognitive Radio channel, imperfect channel state information, estimation errors, worst case.
\end{abstract}

\section{INTRODUCTION}

Cognitive radio (CR) is a highly promising paradigm to provide possible solutions to the spectrum insufficiency problem [1]. To achieve higher spectrum efficiency, the secondary users (SUs) are allowed to share the frequency spectrum with the primary users (PUs) as long as the total interference generated by SUs is below the threshold that the PUs tolerate.

The resource allocation problem has been studied under the assumption of a perfect knowledge of the Channel State Information (CSI) [2]-[7]. However, it is not realistic to assume that the SU has perfect CSI knowledge of its interfering links to the PUs. Indeed, SU may obtain knowledge of its direct link either when the channel reciprocity property is met and/or by some feedback from its receiver. But the PRs cannot be assumed to provide such feedback to the SU. In this context, the problem of robust power allocation in $\mathrm{CR}$ systems has been an area of active research [8]-[14]. The closest work

This work was done during the first author's Ph.D at ETIS laboratory/ UMR 8051, University Paris Seine, University Cergy-Pontoise, ENSEA, CNRS, France. to ours is [10] in which the authors formulate the design of the SU network, where the SUs compete with each other over the resources made available by the PUs, by maximizing their own information rates subject to the transmit power and robust interference constraints in $\mathrm{CR}$ systems in either singleinput single-output (SISO) frequency-selective channels or multiple-input multiple-output (MIMO) channels. Following the philosophy of the worst-case robustness, the problem is formulated by explicitly taking into account the estimation errors of the interfering links SU-to-PU which translates into modified maximum interference constraints that are robust in the worst channel estimation errors. This problem is different and no longer convex as compared with the perfect CSI case in [4].

In this paper, our goal is different than the one in [10]. More precisely, we investigate the impact of estimation errors on the power allocation algorithm minimizing the overall power consumption under QoS and CR interference power constraints proposed in [5], [7] in the case of perfect CSI. The major difference with [10] is that here we do not change the problem formulation. In our analysis, the robustness issue is analysed by considering the the worst case in terms of interfering channel estimation errors at the secondary transmitter.

Our contributions are:

- Two different error models (simple and more realistic) are investigated.

- A robustness analysis of the iterative water-filling algorithm from the SU perspective is provided.

- The impact of these estimation errors at the ST level on the interference caused to the PUs is also analyzed.

- Our analysis shows that the water-filling algorithm not only provides robustness in terms of power consumption but also in terms of scheduling of the SU given the realistic estimation error models especially when the SU is overestimating the interfering power gains.

The paper is organized as follows : In Section II, the system model and estimation error assumptions are introduced. In Section III, we describe the optimization problem and the water-filling algorithm in the perfect CSI case. In Section IV, we analyze robustness issues to estimation errors and we conclude the paper in Section V. 


\section{SYSTEM AND ERROR MODEL}

\section{A. CR channel model}

We focus on the CR channel model in Fig. 1 composed of one secondary user and several $(K \geq 1)$ primary users [7]. Each primary/secondary user consists of a Primary/Secondary Transmitter (PT/ST) and a Primary/Secondary Receiver (PR/SR) respectively. Each device is equipped with only one antenna. The transmission is performed over $N$ orthogonal frequency bands. The transmit power of the ST in the frequency band $n \in\{1, \ldots, N\}$ is denoted by $p_{n}$ and the overall power allocation profile is denoted by $\underline{p}=\left(p_{1}, p_{2}, \ldots, p_{N}\right), \underline{p} \in \mathbb{R}_{+}^{N}$.

\section{Band $n$}

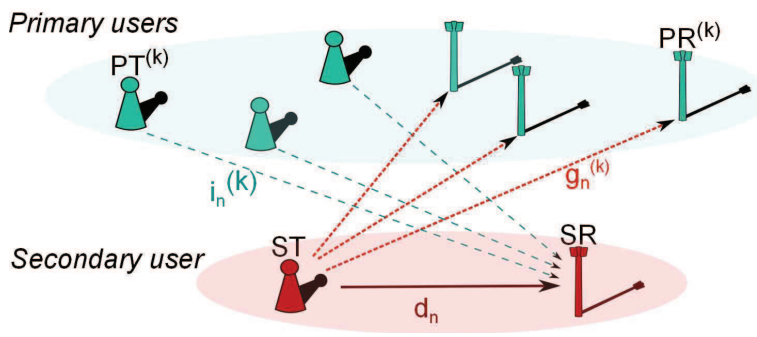

Fig. 1. System model in frequency sub-band $n$.

The received signal at the $\mathrm{SR}$ in band $n$ can be written as:

$$
y_{n}=\sqrt{p_{n} d_{n}} s_{n}+\sum_{k=1}^{K} i_{n}^{(k)}+b_{n},
$$

where $s_{n}$ denotes the transmitted signal at the ST. The instantaneous power gains of the ST-SR direct link and the interfering ST-PR of the $k^{\text {th }}$ PU link are denoted by $d_{n}$ and $g_{n}^{(k)}$ respectively, $k \in \mathcal{K} \triangleq\{1, \ldots, K\}$. All links are assumed to be stationary, ergodic and independent from the noises. The noise in band $n, b_{n} \backsim \mathcal{C N}\left(0, \sigma_{n}^{2}\right)$ is a zero-mean circularly symmetric complex Gaussian variable and the interfering signal from $P T^{(k)}$ is denoted by $i_{n}^{(k)} \backsim \mathcal{C N}\left(0,\left(\tau_{n}^{(k)}\right)^{2}\right)$. The Gaussian assumptions are quite standard for efficient resource allocation in CR channels related works [4], [7], [11]. In this scenario, the Shannon achievable rate of the SU transmission is given by:

$$
R(\underline{p})=\sum_{n=1}^{N} \log _{2}\left(1+c_{n} p_{n}\right)
$$

where $c_{n}$ is the Signal to Interference Noise Ratio (SINR) of the direct link ST-SR and is given by $c_{n}=d_{n} /\left(\sigma_{n}^{2}+\right.$ $\left.\sum_{k=1}^{K}\left(\tau_{n}^{(k)}\right)^{2}\right)$ where $\sum_{k}\left(\tau_{n}^{(k)}\right)^{2}$ is the overall interference power at the PR.

\footnotetext{
${ }^{1}$ The following notations are used in this paper: $|$.$| denotes the absolute$ value, $E($.$) denotes the statistical expectation, \max (x, y)$ and $\min (x, y)$ returns the maximum value and the minimum value of $(x, y)$ respectively, $(.)^{+}$means $\max (0,),. \log _{2}($.$) denotes the logarithm binaire value , \ln ($. denotes the natural logarithm value, $e=\exp (1)$ is the base of natura logarithm and $\Re($.$) denotes the real part of a complex variable.$
}

\section{B. CSI at the ST level}

In the sequel, we assume that the ST has only a perfect knowledge of its own SINR $c_{n}$ in each band. However, the ST does not know the exact power gain $g_{n}^{(k)}$ coming from each PR and knows the estimated one $\widehat{g}_{n}^{(k)}$. We assume that the PRs are sufficiently spaced, i.e., the power gains for all PRs in each band $\left(g_{n}^{(1)}, g_{n}^{(2)}, \ldots, g_{n}^{(K)}\right)$ are independent. Also, we assume that the power gains at the $k^{t h} \mathrm{PR}$ in all orthogonal frequency bands $\left(g_{1}^{(k)}, g_{2}^{(k)}, \ldots, g_{N}^{(k)}\right)$ are independent from those of the $k^{\prime t h} \mathrm{PR}, k \neq k^{\prime}$.

Based on the additive model error of the channel gain proposed in [10] and [8], where the channel estimation error perturbing the channel gain $\epsilon \backsim \mathcal{C N}\left(0, \sigma_{\epsilon}^{2}\right)$ is a zero-mean circularly symmetric complex Gaussian variable, the estimated channel is written as:

$$
\widehat{h}_{n}^{(k)}=h_{n}^{(k)}+\epsilon_{n}^{(k)}
$$

Given that the power gain $g_{n}^{(k)}=\left|h_{n}^{(k)}\right|^{2}$, we consider two different error models perturbing the power gain in each band for each PR:

- Realistic error model: in which we can write the estimated power gain as follows:

$$
\widehat{g}_{n}^{(k)}=g_{n}^{(k)}+e r_{n}^{(k)}, \forall n, \forall k
$$

where, this realistic error $e r_{n}^{(k)}=\left|\epsilon_{n}^{(k)}\right|^{2}+2 \Re\left(h_{n}^{(k)} \epsilon_{n}^{(k)}\right)$ has a mean $\mathbb{E}[e r]=\sigma_{\epsilon}^{2}$ and a variance $\sigma_{e r}^{2}=\sigma_{\epsilon}^{4}+$ $4 g_{n}^{(k)} \sigma_{\epsilon}^{2}$. In most cases of empirical rule, this realistic error model $\mathrm{er}$ is located in the interval $\mathcal{I}_{r}$ depending on its mean $\mathbb{E}[e r]$ and its variance $\sigma_{e r}^{2}$ such that $\mathcal{I}_{r}=$ $\left[\mathbb{E}[e r]-3 \sigma_{e r}^{2} ; \mathbb{E}[e r]+3 \sigma_{e r}^{2}\right]$

- Simple error model: in which we assume independence between the channel gain $h_{n}^{(k)}$ and its error $\epsilon_{n}^{(k)}$, i.e., $2 \Re\left(h_{n}^{(k)} \epsilon_{n}^{(k)}\right) \ll\left|\epsilon_{n}^{(k)}\right|^{2}$, thus, the estimated power gain can be written as follows:

$$
\widehat{g}_{n}^{(k)}=g_{n}^{(k)}+e s_{n}^{(k)}, \forall n, \forall k
$$

where, this simple error $e s_{n}^{(k)}=\left|\epsilon_{n}^{(k)}\right|^{2}$ is an exponentially distributed random variable which has a mean $\mathbb{E}[e s]=\sigma_{\epsilon}^{2}$. In this exponential distribution, this simple error es is located in the interval $\mathcal{I}_{s}$ depending on its mean $\mathbb{E}[e s]$ such that $\mathcal{I}_{s}=$ $[0 ; \ln (4) \mathbb{E}[e s]+1.5 \ln (3) \mathbb{E}[e s]] \simeq\left[0 ; 3 \sigma_{\epsilon}^{2}\right]$.

\section{POWER MINIMIZATION PROBLEM WITH PERFECT CSI}

For the sake of self-containment, we will consider the power minimization problem at the SU level under minimum QoS constraint and maximum interference constraints assuming global perfect CSI at the ST.

\section{A. The Optimization problem and the feasible set}

Given a minimal QoS constraint at the ST and the presence of the PRs, we describe the overall power minimization at the ST level. Similarly to [2], [5], [7], three constraints are considered in this optimization problem the QoS target rate 
constraint; peak and average interference constraints to protect each PR from the interference caused by the ST.

1) Target rate constraint:

$$
R(\underline{p})=\sum_{n=1}^{N} \log _{2}\left(1+c_{n} p_{n}\right) \geq R_{\text {min }},
$$

to achieve a minimum QoS for the ST transmission.

2) Average interference power shaping constraint:

$$
\sum_{n=1}^{N} g_{n}^{(k)} p_{n} \leq \bar{P}^{(k)}, \quad \forall k \in \mathcal{K}
$$

where $\bar{P}^{(k)}$ is the maximum average interference level that can be received at $k^{\text {th }} \mathrm{PR}$.

3) Peak interference power shaping constraints:

$$
0 \leq g_{m}^{(k)} p_{m} \leq P_{m}^{\text {peak }(k)}, \forall k \in \mathcal{K}, m \in \mathcal{N}
$$

where $P_{m}^{\text {peak }(k)}$ is the maximum peak interference level that can be received at $k^{t h} \mathrm{PR}$ in band $m$.

The objective function of minimizing the overall transmit power is in coherence with the CR green communications paradigm. However, the QoS constraint at the ST might impose a minimal transmit power which in turn creates an interference level that is not acceptable by the PR. Thus, the question here is whether the QoS constraint and PR interference constraint (which are opposing constraints) are feasible simultaneously or not. We denote the feasible set by $\mathcal{F}$ such that:

$\mathcal{F}=\left\{\begin{array}{l}\underline{p} \in \mathbb{R}_{+}^{N} \mid 0 \leq g_{m}^{(k)} p_{m} \leq P_{m}^{\text {peak }(k)}, \forall k, \forall m, \\ \sum_{n=1}^{N} \log _{2}\left(1+c_{n} p_{n}\right) \geq R_{\min } ; \sum_{n=1}^{N} g_{n}^{(k)} p_{n} \leq \bar{P}^{(k)}\end{array}\right\}$

The optimization problem is given as follows:

$$
\begin{cases}\text { minimize } & \sum_{n=1}^{N} p_{n} \\ \text { subject to } & \underline{p} \in \mathcal{F}\end{cases}
$$

When $\mathcal{F}$ is non void (see discussion in our work [7]), the optimal power allocation policy is given by a water-filling type of solution. This result follows from the fact that, the problem in (9) is a convex optimization problem [15]. The KKT optimality conditions are both necessary and sufficient in this case. The optimal solution can be computed numerically via a fixed point algorithm or by sub-gradient methods as in [5].

\section{B. Iterative Algorithm based on the estimation at the ST level}

Based on the characterization in the previous subsection, we present a special case of the iterative algorithm, which has been proposed in [7] for the more general case of several secondary users, essssw to compute the solution of the problem (9). To compute the solution to the classical water-filling problem [15] several works in the communications literature (e.g., [16], [17] and references therein) have proposed a finite and deterministic numerical procedure. However, in this problem, the presence of the PUs imposes additional average type constraints (aside the classical minimum QoS constraint for the secondary communication). These additional constraints are the main reason for which the classical numerical approach fails. Instead, we present the fixed-point iterative algorithm that converges to one of the optimal solutions which was proposed in [7] and which is similar but not equivalent to the sub-gradient method in [5]. It turns out that the convergence

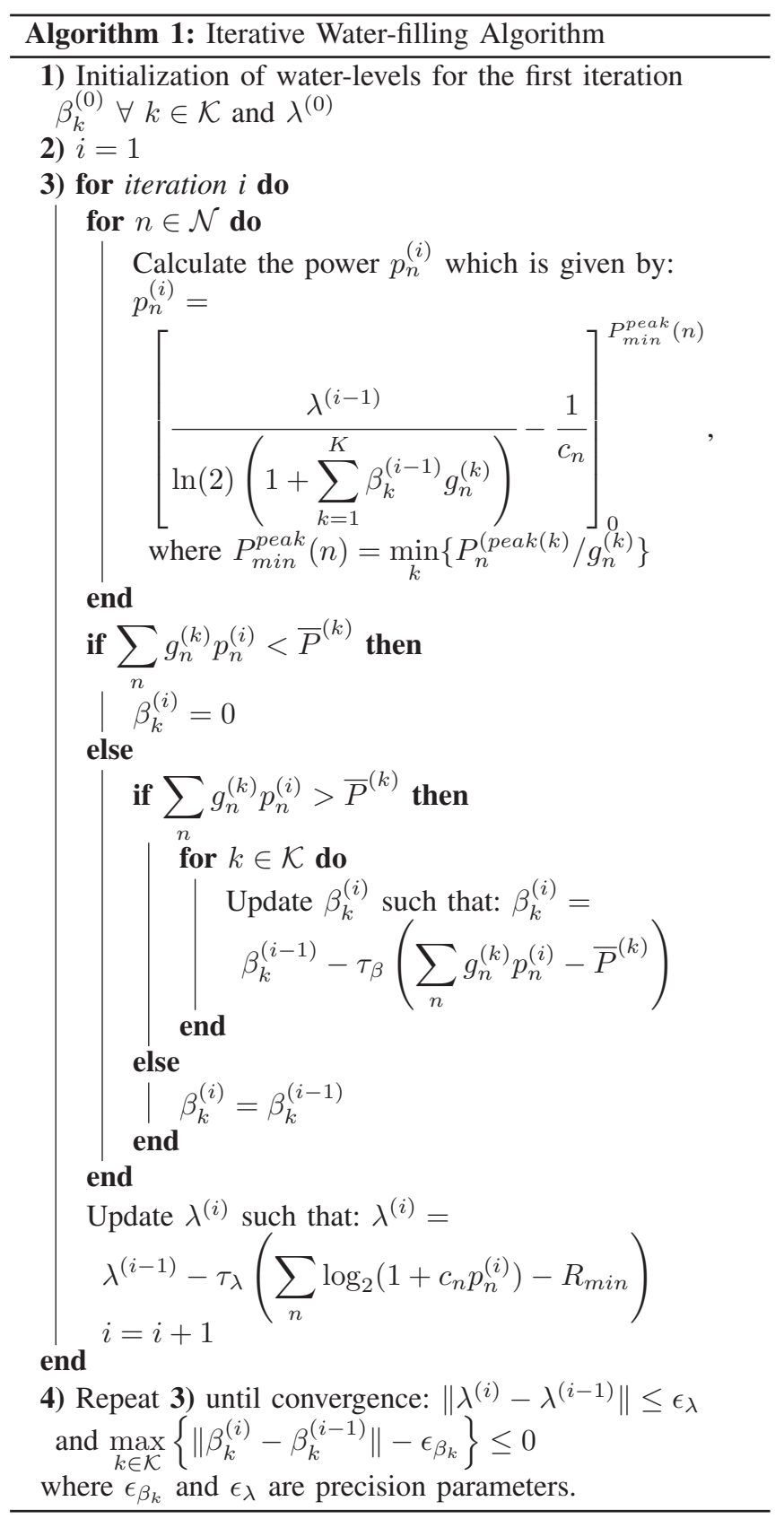

point is an optimal solution to the general problem in (9) provided that an optimal solution exists.

Proposition 1: Whenever Algorithm 1 converges, then the 
convergence point is an optimal point of the general minimization problem.

The proof of this proposition is based on convex optimization tools is discussed in details in [7]. The intuition is that, at the convergence state, the iteration is satisfying the KKT conditions which are both necessary and sufficient for optimality of the convex optimization problem (9). We have validated the convergence of the proposed algorithm via extensive MonteCarlo simulations. Also, our algorithm is very similar to the sub-gradient method [5] based on dual Lagrange function, the proof of convergence is similar too.

\section{ROBUSTNESS ANALYSIS}

In this Section, we will consider the case of imperfect CSI at the ST level, as described in Section II, in order to analyze the robustness of our iterative water-filling algorithm for our power allocation problem to the channel estimation errors.

\section{A. Worst-Case Estimation Errors}

In this subsection, we study the performance of Algorithm 1. We assume a more realistic scenario in which the SU doesn't know the true power gain $g_{n}^{(k)}$ but only an estimation. We study the robustness based on the worst estimation error. Two cases can be distinguished in function of the error: i) overestimation, and ii) under-estimation errors.

1) Over-estimation of a power gain: $g_{n}^{(k)}<\widehat{g}_{n}^{(k)}$, implies that the estimated feasible set $\widehat{\mathcal{F}}$ is smaller than the true feasible set $\mathcal{F}$, i.e., $\widehat{\mathcal{F}} \subseteq \mathcal{F}$. This means that the true interference constraints at the PUs are always satisfied by the SU. Thus, the major focus here is on the SU loss of performance. For this reason, we consider the worst estimation errors that affect the SU performance. This is the case in which all interfering channels are overestimated at the maximum value. Under the hypothesis in subsection II-B and considering that the variance of the error $\epsilon_{n}^{(k)}$ in the channel gain is negligible compared to the actual power gain i.e., $\sigma_{\epsilon}^{2} \ll g_{n}^{(k)}$, our error models in the worst case can be given as follows:

- Realistic error model: Using the realistic error model in (4), we can write the worst case estimated power gain as follows:

$$
\widehat{g}_{n}^{(k), \text { Worse }} \simeq g_{n}^{(k)}+12 g_{n}^{(k)} \sigma_{\epsilon}^{2}
$$

where the maximum value of the realistic error multiplied with the gain power is coming from the interval $\mathcal{I}_{r}$ in which this error is located and assuming that $\sigma_{\epsilon}^{2} \ll g_{n}^{(k)}$ such that we add $\mathbb{E}[e r]+3 \sigma_{e r}^{2}=\sigma_{\epsilon}^{2}+3\left(\sigma_{\epsilon}^{4}+4 g_{n}^{(k)} \sigma_{\epsilon}^{2}\right) \simeq$ $12 g_{n}^{(k)} \sigma_{\epsilon}^{2}$ to the actual gain in the worst case.

- Simple error model: Using the simple error model in (5), we can write the worst case estimated power gain as follows:

$$
\widehat{g}_{n}^{(k), \text { Worse }} \simeq g_{n}^{(k)}+3 \sigma_{\epsilon}^{2}
$$

where the maximum value of the simple error added to the gain power is coming from the interval $\mathcal{I}_{s}$ in which this error is located and assuming that $\sigma_{\epsilon}^{2} \ll g_{n}^{(k)}$ such that we add $+3 \sigma_{\epsilon}^{2}$ to the actual gain in the worst case.
2) Under-estimation of a true power gain: $g_{n}^{(k)}>\widehat{g}_{n}^{(k)}$, implies an estimated feasible set $\widehat{\mathcal{F}}$ larger than the actual feasible set $\mathcal{F}$, i.e., $\mathcal{F} \subseteq \widehat{\mathcal{F}}$. This means that the performance of the $\mathrm{SU}$ given this estimation error is improved. The major issue is that the SU takes advantage of the less restrictive estimated interference constraints. This in turn, creates interference to the PUs which may be above the true tolerated values. Thus, the focus is no longer on SU performance but on the extent at which the SU may violate the true interference constraints imposed by the PU. For this reason, we consider the estimation errors that affect the most the interference created to the PU. This is the case in which all interfering channels are underestimated at the minimum value. Under the hypothesis in subsection II-B and considering that $\sigma_{\epsilon}^{2} \ll g_{n}^{(k)}$, our error models in the worst case can be given as follows:

- Realistic error model: In the worst case the power gain can be underestimated as follows:

$$
\widehat{g}_{n}^{(k), \text { Worse }} \simeq g_{n}^{(k)}-12 g_{n}^{(k)} \sigma_{\epsilon}^{2}
$$

where the minimum value of the realistic error multiplied with the gain power is coming from the minimum value in the interval $\mathcal{I}_{r}$ and assuming that $\sigma_{\epsilon}^{2} \ll g_{n}^{(k)}$ such that we add $\mathbb{E}[e r]-3 \sigma_{e r}^{2}=\sigma_{\epsilon}^{2}-3\left(\sigma_{\epsilon}^{4}+4 g_{n}^{(k)} \sigma_{\epsilon}^{2}\right) \simeq-12 g_{n}^{(k)} \sigma_{\epsilon}^{2}$ to the actual gain in the worst case.

- Simple error model: We have seen that, in this model, we can not underestimate the power gain as the minimum value added to the gain power coming from the minimum value in the interval $\mathcal{I}_{s}$ is null in the worst case, i.e., $\widehat{g}_{n}^{(k), \text { Worse }} \simeq g_{n}^{(k)}$.

\section{B. Numerical Simulations}

In this Section, we analyze the impact of the channel estimation errors on the aforementioned issues (on power consumption at the SU and on the maximum interference inflicted on the PUs) via numerical simulations. We illustrate some of the most representative results by focusing on the specific case where only two orthogonal frequency bands are available $N=2$. However, our analysis and observations carry over the general case which will not be illustrated because of space limitations and also higher dimensional feasible sets which are harder or even impossible to visualise.

1) Over-estimation errors: We start by discussing the case in which the channel power gain estimations are larger than the actual power gains of the interfering links from SU to PUs. In this case, the estimation errors have no impact on the PUs and the interference constraints are guaranteed. Thus, we are interested to evaluate the SU performance reduction caused by the worst case errors in function of the error variance $\sigma_{\epsilon}^{2}$.

In Fig. 2 and Fig. 3, we illustrate the scenario $N=2$ frequency bands and $K=3$ primary users with the system parameters $^{1}: R_{\text {min }}=4, c_{1}=1.5, c_{2}=2.4, \vec{P}_{1}^{\text {peak }}=\left[\begin{array}{lll}5 & 6 & 7\end{array}\right]$, $\vec{P}_{2}^{\text {peak }}=\left[\begin{array}{lll}5 & 5 & 7\end{array}\right]$ and $\bar{P}^{(k)}=10, \widehat{g}_{1}^{(k)}=1, \widehat{g}_{2}^{(k)}=0.1, \forall k$.

${ }^{1}$ We denote by $\vec{x}$ the $\mathrm{K}$ dimensional vector containing all the quantities $x^{(k)}$ in PU $k \in \mathcal{K}: \vec{x} \triangleq\left[x^{(1)}, \ldots, x^{(K)}\right]$ 


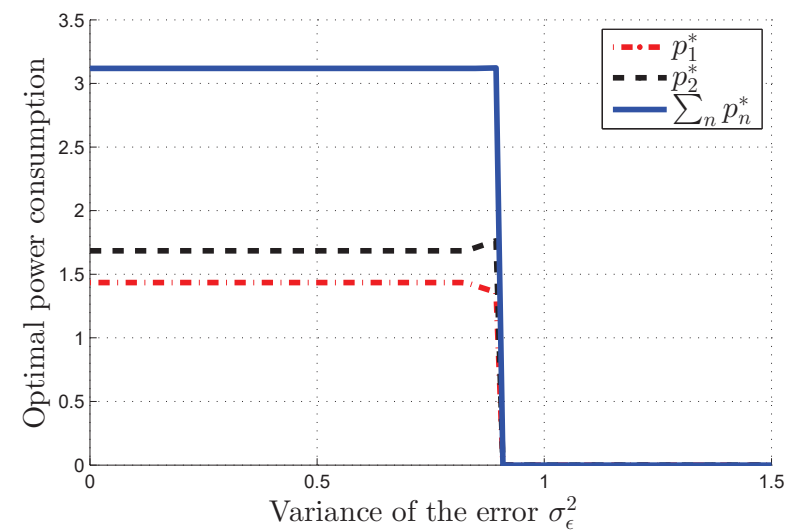

(a) Power consumption vs. the variance of the error $\sigma_{\epsilon}^{2}$
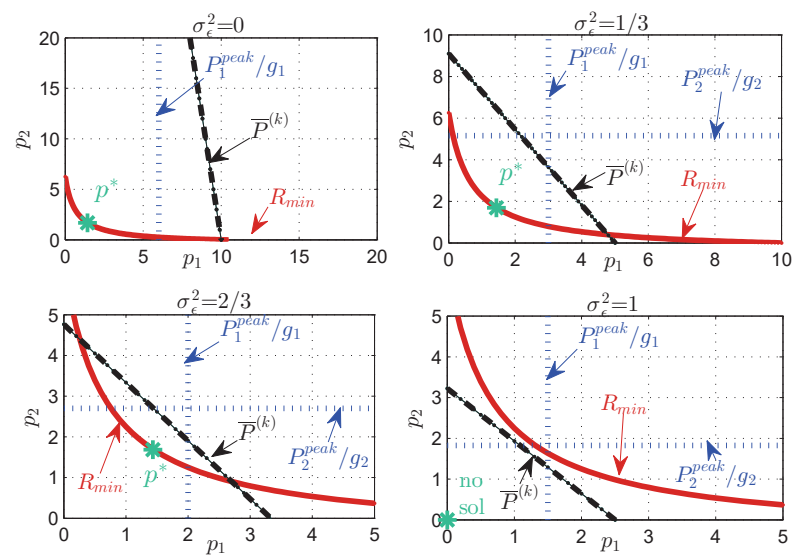

(b) Feasible set $\widehat{\mathcal{F}}$ : QoS and CR interference power constraints

Fig. 2. Overestimation assuming the simple error model.

In Fig. 2, we investigate the simplified error model. Notice that the optimal power consumption $\left(\sum_{n} p_{n}^{*}\right)$ when there are no estimation errors (i.e., $\sigma_{\epsilon}^{2}=0$ ) is identical to the perfect CSI case. When the error variance increases, we observe in Fig. 2(a) that there is little difference with the perfect CSI case regarding the optimal powers $\left(p_{1}^{*}, p_{2}^{*}\right)$ and the optimal overall power consumption. This means that, assuming the simplified error model is realistic, the SU is robust to estimation errors until a certain threshold on the error variance is attained. This threshold can be explained by analysing Fig. 2(b). As the error variance increases, the estimated feasible set becomes more and more restricted (because of the increasingly restrictive estimated interference constraints) until it becomes void.

In Fig. 3, we investigate the more realistic error model. Fig. 3(a) shows that the behaviour of the optimal power consumption $\left(\sum_{n} p_{n}^{*}\right)$ as function of the error variance is no longer the same. As the error variance increases, the SU performance at the optimal point is decreasing and its power consumption is increasing. This means that, the SU is less robust in terms of power consumption as opposed to the simplified error model case. Similarly to the previous case, there is a threshold on the error variance, above which the estimated feasible set becomes void and the SU is not

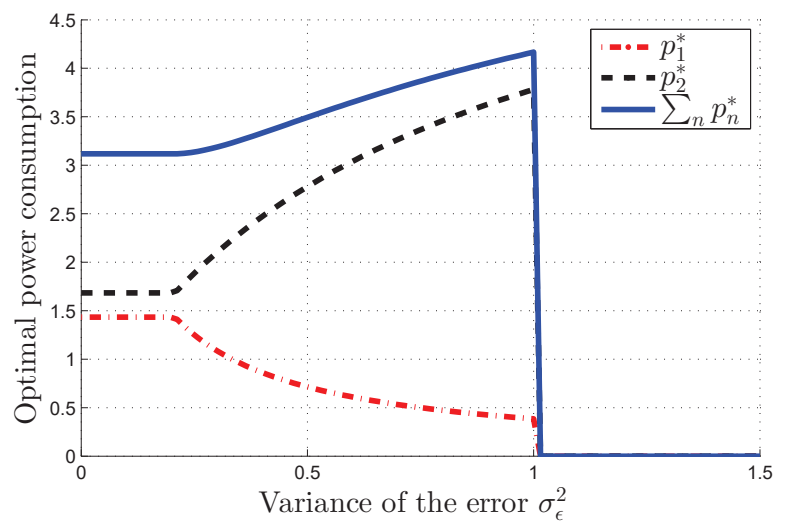

(a) Power consumption vs. the variance of the error $\sigma_{\epsilon}^{2}$
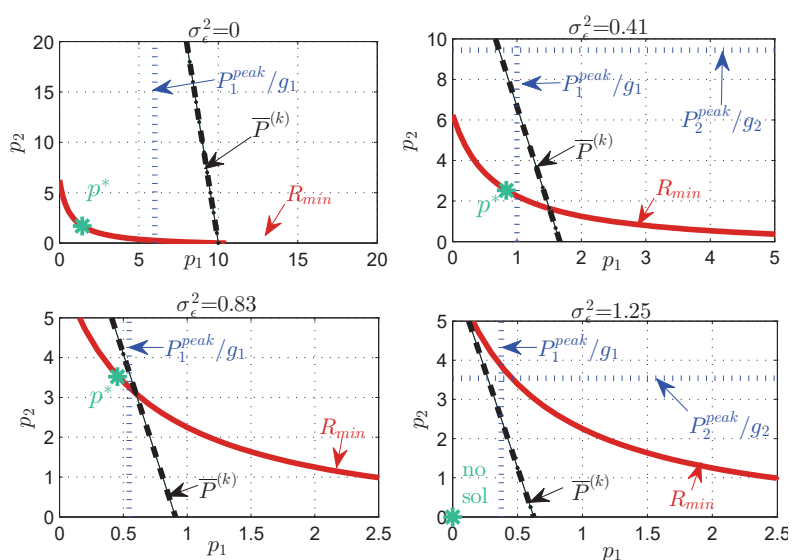

(b) Feasible set $\widehat{\mathcal{F}}$ : QoS and CR interference power constraints Fig. 3. Overestimation assuming the realistic error model.

scheduled in the system. The interesting thing to remark is that this threshold is significantly higher in the realistic error model than the previous one. This means that the SU is more robust in terms of scheduling in this case.

Fig. 3(b) provides the elements to explain this observation. The error model has a direct impact on the shape of the interference constraints. In the simplified model, the estimated constraints change by adding a constant parameter to the power gains (i.e., $3 \sigma_{\epsilon}^{2}$ ) which reduces the power constraints in a symmetric way (see Fig. 2(b)) causing them to fall below the minimum rate constraint faster than the realistic case. Indeed, in the realistic case the added error is not constant but depends on the power gain (i.e., $12 \sigma_{\epsilon}^{2} g_{n}^{(k)}$ ), which causes the power constraints to reduce in a proportional manner w.r.t. the gains making these constraints to fall below the rate curve slower than the simple case.

2) Under-estimation errors: If the SU under-estimates the power gains of its interfering links to the PUs, then the estimated feasible set is larger than the true feasible set and the performance of the SU may even increase as a result of these estimation errors. The robustness of the SU's performance is not the issue here. These errors allow the SU to under-estimate their interference levels caused to the PUs which may result 
in the violation of the true PU constraints. This is a major issue in CR channels in which the primary consideration is guaranteeing the maximum level of interference at the PUs.

First, we remark that for the simplified error model, the added error is an exponentially distributed random variable, and, thus, is always positive. In this case, there can never be any under-estimation errors and the PUs interference constraints are always verified.
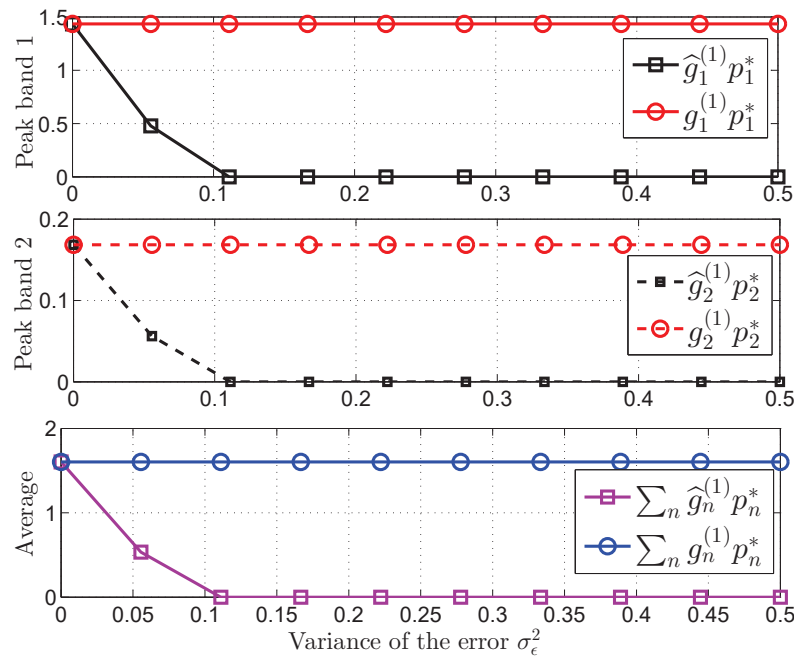

Fig. 4. Under-estimation errors do not necessarily impact on the PUs.

We focus now, on the realistic error model. In Fig. 4, we consider the same scenario as in Fig. 3. We focus on the first PU and we plot the estimated peak and average interference caused by the SU at its optimal solution (computed based on the worst case estimations) and the actual inflicted interference. First, we remark that the optimal solution is not influenced, in this scenario, by the estimation error (i.e., $\left.\sum_{n} g_{n}^{(1)} p_{n}^{*}<\bar{P}^{(1)}=10\right)$. This can be explained by the first of the four plots in Fig. 3(b) illustrating the feasible set and the solution in the perfect CSI case. The solution lays inside of the strict interior of the set defined by the interference constraints. In such a case, it is easy to see that the solution does not change by augmenting the set of interference constraints. This means that, whenever the solution in the perfect CSI case is not near the border of the interference constraints, the underestimation errors have no impact on the optimal solution. Therefore, in such cases neither the SU nor the PUs will be impacted by such errors.

In Fig. 5, we plot the estimated peak and average interference caused by the SU at its optimal solution and the actual inflicted interference, in the following scenario: $N=2, K=1$ (only one primary user), $c=[10,1], g=[10,0.1], \bar{P}=10$, $P^{\text {peak }}=[5,5]$. In such a case, in which a given sub-channel provides a very good direct link to the $\mathrm{SU}\left(c_{1}>>c_{2}\right)$ but also has a critical interfering link to one of the PUs $\left(g_{1}>>g_{2}\right)$ under-estimation errors are critical. We observe that, even for very low error variance values, the actual interference caused to the PU is well-above the tolerated limit: both the average interference constraint and the peak interference constraint
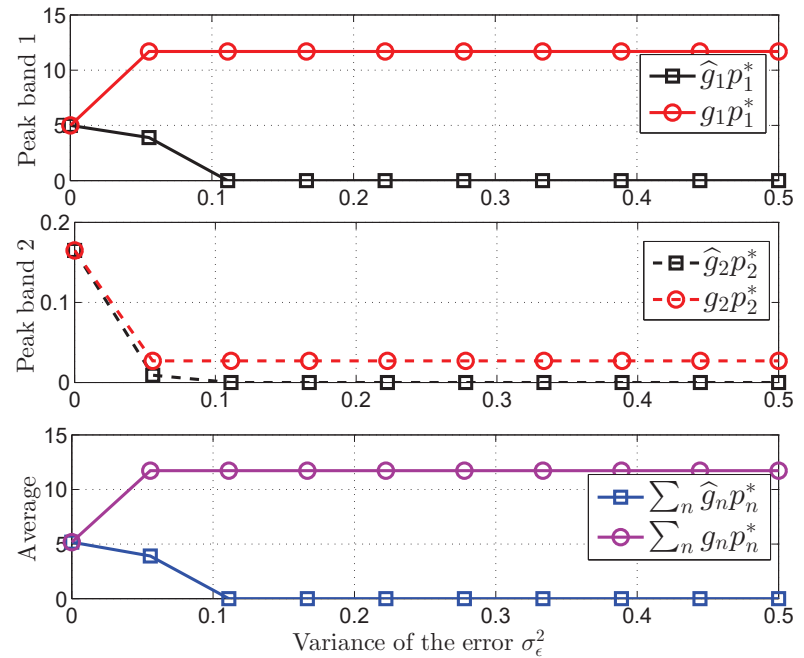

Fig. 5. Under-estimation errors have a crucial impact on the PUs interference constraints.

in the first sub-band are violated by the SU because of these under-estimation errors (i.e., $g_{1} p_{1}^{*}>P^{\text {peak }}=5$ and $\left.\sum_{n} g_{n} p_{n}^{*}>\bar{P}^{(1)}=10\right)$.

Several solutions are possible to cope with this issue. One solution, proposed by [10], consists in incorporating these errors in the problem formulation and entirely change the SU power allocation algorithm. However, such approaches may turn out to be too rigid and may censure the SU which will not be allowed to transmit in most cases. A more simpler solution, based on the analysis here above, is to check whether the optimal power allocation in the perfect CSI case does not lie near the border of the maximal interference constraints. If so, then they transmit based on their estimation, otherwise they are not allowed to transmit. Now the problem is that, of course, the SU does not posses the perfect CSI knowledge. However, if we assume that the SU knows the variance of the estimation error (which is a rather reasonable assumption), then the SU may compute the solution based on the worst case analysis in Section IV-A using the following interfering link power gains:

$$
\tilde{g}_{n}^{(k)}=\frac{\hat{g}_{n}^{(k)}}{1-12 \sigma_{\epsilon}^{2}}, \forall n, \forall k .
$$

If this solution is not near the borders of the interference constraints, then the SU communicates based on his estimation, otherwise, the interference constraints are considered critical and the SU is no longer scheduled. Extensive simulations are needed to further validate this point.

\section{CONCLUSION}

In this paper, we analyzed the impact of imperfect CSI in a cognitive radio channel on the optimal power allocation policy of the secondary user (SU) that minimizes its power consumption under rate and maximal interference to the primary users (PUs) constraints. The worst cases in terms of estimation errors were analyzed from both, the SU and PUs perspective. When the SU is over-estimating its interfering 
links to the PUs, the SU is censuring too much its transmission and thus incurs a loss of performance, which depends on the error model and the error variance. When the $\mathrm{SU}$ is under-estimating its interfering links to the PUs, it is underestimating its transmission effects on the PUs, which may result in interference constraint violations. A simple way to account for this issue was proposed, which has the advantage not to over-censure the SU transmission especially when the $\mathrm{SU}$ is over-estimating the interfering power gains.

\section{REFERENCES}

[1] S. Haykin, "Cognitive radio: brain-empowered wireless communications," Selected Areas in Communications, IEEE Journal on, vol. 23, no. 2, pp. 201-220, 2005.

[2] J.-S. Pang, G. Scutari, F. Facchinei, and C. Wang, "Distributed power allocation with rate constraints in gaussian parallel interference channels," Information Theory, IEEE Transactions on, vol. 54, no. 8, pp. 3471-3489, 2008.

[3] J.-S. Pang, G. Scutari, D. P. Palomar, and F. Facchinei, "Design of cognitive radio systems under temperature-interference constraints: A variational inequality approach," Signal Processing, IEEE Transactions on, vol. 58, no. 6, pp. 3251-3271, 2010.

[4] G. Scutari and D. P. Palomar, "MIMO cognitive radio: A game theoretical approach," Signal Processing, IEEE Transactions on, vol. 58, no. 2, pp. 761-780, 2010

[5] D. T. Ngo and T. Le-Ngoc, "Distributed resource allocation for cognitive radio networks with spectrum-sharing constraints," Vehicular Technology, IEEE Transactions on, vol. 60, no. 7, pp. 3436-3449, 2011.

[6] R. Masmoudi, E. V. Belmega, I. Fijalkow, and N. Sellami, "A closedform solution to the power minimization problem over two orthogonal frequency bands under QoS and cognitive radio interference constraints," in IEEE International Symposium on Dynamic Spectrum Access Networks (DYSPAN), 2012, 2012, pp. 212-222.

[7] R. Masmoudi, E. V. Belmega, and I. Fijalkow, "Efficient spectrum scheduling and power management for opportunistic users," EURASIP Journal on Wireless Communications and Networking (JWCN), vol. 2016, no. 97, pp. 1 - 19, Apr. 2016.

[8] A. Pascual-Iserte, D. Palomar, A. Perez-Neira, and M. Lagunas, "A robust Maximin approach for MIMO communications with imperfect channel state information based on convex optimization," IEEE Trans. on Signal Processing, vol. 54, no. 1, pp. 346 - 360, Jan. 2006.

[9] J. Wang and D. Palomar, "Worst-case robust MIMO transmission with imperfect channel knowledge," IEEE Trans. on Signal Processing, vol. 57, no. 8, pp. 3086 - 3100, Aug. 2009.

[10] J. Wang, G. Scutari, and D. Palomar, "Robust MIMO cognitive radio via game theory," IEEE Trans. on Signal Processing, vol. 59, no. 3, pp. 1183 - 1201, Mar. 2011.

[11] A. Bagayoko, I. Fijalkow, and P. Tortelier, "Power control of spectrumsharing in fading environment with partial channel state information," Signal Processing, IEEE Transactions on, vol. 59, no. 5, pp. 2244-2256, 2011.

[12] Y. Yang, G. Scutari, P. Song, and D. Palomar, "Robust MIMO cognitive radio systems under interference temperature constraints," IEEE Journal on Selected Areas in Communications, vol. 31, no. 11, pp. 2465 - 2482, Nov. 2013.

[13] J. Wang, J. Chen, Y. Lu, M. Gerla, and D. Cabric, "Robust power control under location and channel uncertainty in cognitive radio networks," IEEE Wireless Communications Letters, vol. 4, no. 2, pp. 113 - 116, Dec. 2014.

[14] R. Fan, W. Chen, J. An, F. Gao, and G. Wang, "Robust power and bandwidth allocation in cognitive radio system with uncertain distributional interference channels," IEEE Transactions on Wireless Communications, vol. 15 , no. 10 , pp. $7160-7173$, Oct. 2016.

[15] S. Boyd and L. Vandenberghe, Convex optimization. Cambridge university press, 2004

[16] G. Scutari, D. P. Palomar, and S. Barbarossa, "Asynchronous iterative water-filling for gaussian frequency-selective interference channels," Information Theory, IEEE Transactions on, vol. 54, no. 7, pp. 28682878, 2008.
[17] D. P. Palomar and J. R. Fonollosa, "Practical algorithms for a family of waterfilling solutions," Signal Processing, IEEE Transactions on, vol. 53, no. 2, pp. 686-695, 2005. 